\title{
Desenvolvimento de transtornos mentais relacionados à úlcera venosa: revisão sistemática
}

\author{
Development of mental disorders related to venous ulcer: systematic review
}

Desarrollo de trastornos mentales relacionados con la úlcera venosa: revisión sistemática

Adriana Souza Szpalher ${ }^{1 *}$.

\begin{abstract}
RESUMO
Objetivo: Avaliar o desenvolvimento de transtornos psicológicos relacionados à presença de úlcera venosa de perna (UVP). Métodos: revisão sistemática em julho de 2019 com metodologia de Donato H e Donato $\mathrm{M}$ (2019) e Recomendação PRISMA para responder à questão: há desenvolvimento de transtornos psicológicos relacionados à UVP? Incluídos estudos originais, sem delimitação temporal, em português, inglês e espanhol, nas Bases US National Library of Medicine (PubMed), Biblioteca Virtual de Saúde (BVS), The Cochrane Central Register of Controlled Trials (CENTRAL) abrangendo a Base eletrônica editora Elsevier (EMBASE), Scientific Electronic Library Online (SciELO) e Health Evidence. Resultados: A busca retornou 256 estudos, após análise completa foram excluídos 248 por não atenderem a metodologia e objetivo, resultando em 8 estudos, prevalecendo em inglês. 816 indivíduos foram avaliados, predominando como transtorno mental a depressão. Houve diversidade quanto ao Tipo de estudo. Considerações finais: há pouca produção relacionada à temática e não foi encontrada revisão sistemática. Sugerem-se estudos que aprofundem o impacto dos transtornos psicológicos na UVP, enfatizando os níveis citológico e histológico. Acredita-se que pesquisas futuras trarão reflexões acerca da importância do atendimento psicológico e psiquiátrico, associado à promoção de atividades de bem-estar e do convívio social.
\end{abstract}

Palavras-chave: Transtornos Mentais, Saúde Mental, Úlcera da Perna.

\begin{abstract}
Objective: To evaluate the development of psychological disorders related to the presence of venous leg ulcer (VLU). Methods: Systematic review in July 2019 with Donato H and Donato M methodology (2019) and PRISMA Recommendation to answer the question: Is there a development of psychological disorders related to VLU? Included original, non-time-bound studies in Portuguese, English, and Spanish in databases US National Library of Medicine (PubMed), Virtual Healthcare Library (VHL), The Cochrane Central Register of Controlled Trials (CENTRAL) covering the Elsevier Publishing Database (EMBASE), Scientific Electronic Library Online (SciELO) and Health Evidence. Results: The search returned 256 studies, after complete analysis 248 were excluded because they did not meet the methodology and objective, resulting in 8 studies, prevailing in English. 816 individuals were evaluated, predominating as mental disorder depression. There was diversity regarding the type of study. Final considerations: there is little production related to the theme and no systematic review was found. Studies are suggested to deepen the impact of psychological disorders on VLU, emphasizing the cytological and histological levels. It is believed that future research will bring reflections on the importance of psychological and psychiatric care, associated with the promotion of welfare activities and social life.
\end{abstract}

Key words: Mental Disorders, Mental Health, Leg Ulcer.

\section{RESUMEN}

Objetivo: evaluar el desarrollo de trastornos psicológicos relacionados con presencia de úlcera venosa de la pierna (UVP). Métodos: Revisión sistemática en julio de 2019 con metodología de Donato $\mathrm{H}$ y Donato $\mathrm{M}$ (2019) y Recomendación PRISMA para responder la pregunta: ¿hay desarrollo de trastornos psicológicos relacionados con la UVP? Se incluyeron estudios originales sin límite de tiempo en portugués, inglés y español en US National Library of Medicine (PubMed), Biblioteca Virtual en Salud (BVS), The Cochrane Central

1Universidade Federal do Estado do Rio de Janeiro (UNIRIO), Rio de Janeiro-RJ. *E-mail: adrisz88@hotmail.com 
Register of Controlled Trials (CENTRAL) cubriendo la Base de Publicaciones de Elsevier (EMBASE), Scientific Electronic Library Online (SciELO) y Health Evidence. Resultados: búsqueda arrojó 256 estudios, luego de análisis completo, se excluyeron 248 porque no cumplían con la metodología y objetivo; resultó en 8 estudios, prevaleciente en inglés. Se evaluaron 816 individuos, predominando la depresión como trastorno mental. Hubo diversidad con respecto al tipo de estudio. Consideraciones finales: hay poca producción relacionada con el tema; no se encontró revisión sistemática. Se sugieren estudios para profundizar el impacto de trastornos psicológicos en la UVP, enfatizando niveles citológico y histológico. Se cree que la investigación futura traerá reflexiones sobre importancia de atención psicológica y psiquiátrica, asociada con promoción de actividades de bienestar y vida social.

Palabras clave: Trastornos Mentales, Salud Mental, Úlcera de la Pierna.

\section{INTRODUÇÃO}

A úlcera venosa de perna (UVP) é visto como um problema de saúde pública com importante impacto socioeconômico e é uma das maiores causas de mortalidade e morbidade em nível global, principalmente por estar associada a fatores sistêmicos, ocasionando, assim, sua cronicidade, recorrentes episódios e dificuldade de cicatrização. As lesões provenientes da UVP resultam em dor e limitação, afetando as atividades cotidianas e a qualidade de vida (QV) (SALOMÉ GM, et al., 2015).

Os portadores de UVP passam por diversas mudanças como a mobilidade diminuída, odor e exsudato provenientes da ferida, alteração física das pernas, perda de autonomia e comprometimento de atividades, inclusive as relacionadas ao lazer e à interação social. Consequentemente, estas mudanças têm um impacto negativo na autoestima, emocional, física, espiritual e, posteriormente, o desenvolvimento de desordens mentais como altos níveis de depressão, alterações de sono, abuso de substâncias entre outros (KOURIS A, et al., 2014; SALOMÉ GM, et al., 2015). Nesse sentido, Walburn J et al. (2017) citam a importante relação entre a deficiente cicatrização de UVP e emoções negativas.

Kouris A, et al. (2014) e Platsidaki E, et al. (2017) lembram que o status psicológico se altera conforme o aumento da complexidade da doença, trazendo sentimentos como raiva, constrangimento, distorção da imagem corporal, isolamento social e ansiedade em alto grau por conta de implicações no âmbito laboral e conjugal, por exemplo. O prejuízo na QV reflete diretamente no impacto psicossocial destes indivíduos; e apontam que a QV de portadores de UVP é amplamente estudada, porém, há escassa produção de estudos que abordem a presença de transtornos psicológicos nos portadores de UVP.

Romic I et al. (2015) apresentam o resultado de seu estudo sobre QV, no qual conclui que o impacto da UVP no domínio saúde mental é altamente considerável; lembram que esta mazela traz efeitos multidimensionais, manifestados tanto na esfera física quanto na psicológica, trazendo sentimentos como o de inutilidade. Ainda mencionam os efeitos indiretos que podem comprometer o indivíduo em nível psicológico, como a alta dependência de outros.

Araújo RO et al. (2016) identificaram, em seu estudo sobre QV, associação estatística significativa entre tempo de existência de UVP e estado emocional, com predomínio naqueles com a patologia há mais de um ano. Ressaltam a possibilidade de alterações na saúde mental prejudicada por fatores geralmente físicos, como o desconforto pela presença de um curativo. Ainda sugerem, como estratégia para enfrentamento das consequências da UVP, assistência profissional no sentido de preparar o indivíduo para lidar com o tratamento proposto.

Ressalta-se que após extensa busca em bases de dados eletrônicas, não foram encontradas revisões (seja ela integrativa ou sistemática) que abordem a temática, apenas estudos nos quais abordam o impacto da UVP na QV.

Diante do exposto, este estudo justifica-se pela carência de publicações, compreendendo, assim, a necessidade em aprofundar tal assunto. Assim, teve-se como Objetivo avaliar o desenvolvimento de transtornos psicológicos relacionados à presença de úlcera venosa em membros inferiores. 


\section{MÉTODOS}

Trata-se de uma revisão sistemática (RS) -quantitativo e retrospectivo-, tendo como referencial teórico os critérios de investigação sistemática conforme Donato $H$ e Donato $M$ (2019): formulação da questão de investigação; produção de um protocolo de investigação que contemple os critérios de inclusão e exclusão, a estratégia de pesquisa, seleção e avaliação qualitativa dos estudos, extração e síntese dos dados e, por último, a publicação da pesquisa. Foi utilizado o checklist do Preferred Reporting Items for Systematic Reviews and Meta-Analyses (PRISMA) para a produção dos tópicos/seções desta pesquisa. A pesquisa ocorreu no mês de julho de 2019 e adotou-se a questão de investigação: há desenvolvimento de transtornos psicológicos relacionados à úlcera venosa de perna?, sendo utilizada a estratégia que representa 0 acrônimo para Problema, Intervenção, Comparação, Resultado e Tipo de estudo (PICOS) para formulação da questão de investigação, conforme recomendação do PRISMA, no qual incluiu o Problema (problem), Resultado (outcomes) e Tipo de estudo (study design).

Os critérios de inclusão são: estudos originais do tipo Observacional (caso controle, Coorte, seccional), Descritivo (relato de caso, prevalência, série de casos), quantitativos ou qualitativos, publicados em revistas científicas e completos, relacionados ao Objeto de estudo, em português, inglês e espanhol. Ressalta-se que não foi encontrada publicação de RS com o Objetivo presente neste estudo, logo, não se adotou delimitação temporal intencionando-se, assim, resgatar a maior quantidade de publicações para responder à questão de investigação, conforme recomendação em literatura (DONATO H e DONATO M, 2019). Ausência de grupo comparação não foi critério de exclusão.

Foram utilizados Descritores em ciências da saúde (DeCS) em português: Transtornos mentais, Estresse psicológico, Saúde mental, Úlcera da perna, Úlcera e Úlcera varicosa, e seus derivados em inglês e espanhol, sendo combinados com operadores booleanos AND, OR e NOT, este último com intuito de excluir artigos relacionados a outras enfermidades identificadas durante a busca (Quadro 1).

Quadro 1 - Bases de dados e estratégia de busca utilizada.

("Mental Disorders"[All Fields] OR "Stress Psychological"[All Fields] OR "Mental Health"[All Fields]) AND ("Leg Ulcer"[All Fields] OR "Ulcer"[All Fields] OR "Varicose Ulcer"[All Fields]) NOT (("obesity"[MeSH Terms] OR "obesity"[All Fields]) OR Diabetic[All Fields] OR Colorectal[All Fields] OR ("stomach"[MeSH Terms] OR $\begin{array}{ll}\text { PubMed } & \text { "stomach"[All Fields] OR "gastric"[All Fields]) OR gastrointestinal[All Fields] OR peptic[All Fields] OR } \\ \text { ("neoplasms"[MeSH Terms] OR "neoplasms"[All Fields] OR "cancer"[All Fields]) OR ("heart"[MeSH Terms] }\end{array}$ OR "heart"[All Fields] OR "coronary"[All Fields]) OR renal[All Fields] OR spinal[All Fields] OR ("pressure"[MeSH Terms] OR "pressure"[All Fields]) OR ("duodenum"[MeSH Terms] OR "duodenum"[All Fields] OR "duodenal"[All Fields]))

("Mental Disorders" OR "Stress Psychological" OR "Mental Health" OR “Trastornos Mentales" OR Portal "Transtornos Mentais" OR "Estrés Psicológico" OR "Estresse Psicológico" OR "Salud Mental" OR "Saúde BVS Mental") AND ("Leg Ulcer" OR "Ulcer" OR "Úlcera" OR "Varicose Ulcer" OR "Úlcera de la Pierna" OR "Úlcera da Perna" OR "Úlcera Varicosa") NOT (Obesity OR Diabetic OR Colorectal OR gastric OR gastrointestinal OR peptic OR cancer OR coronary OR renal OR spinal)

("Mental Disorders" OR "Stress Psychological" OR "Mental Health" OR “Trastornos Mentales" OR CENTRAL "Transtornos Mentais" OR “Estrés Psicológico" OR "Estresse Psicológico" OR “Salud Mental” OR "Saúde EMBASE "Úlcera da Perna" OR "Úlcera Varicosa") NOT (Obesity OR Diabetic OR gastric OR peptic OR cancer OR coronary OR renal OR spinal OR colorectal OR gastrointestinal)

("Mental Disorders" OR "Stress Psychological" OR "Mental Health" OR "Trastornos Mentales" OR "Transtornos Mentais" OR "Estrés Psicológico" OR "Estresse Psicológico" OR "Salud Mental" OR "Saúde

SciELO Mental") AND ("Leg Ulcer" OR "Ulcer" OR "Úlcera" OR "Varicose Ulcer" OR "Úlcera de la Pierna" OR "Úlcera da Perna" OR “Úlcera Varicosa") NOT (Obesity OR Diabetic OR gastric OR peptic OR cancer OR coronary OR renal OR spinal OR colorectal OR gastrointestinal)

\section{Health "Mental health" AND Ulcer \\ Evidence Psychological AND ulcer}

Fonte: Szpalher AS, 2019.

Foram excluídos os artigos incompletos, não relacionados ao Objeto estudado, relatos de experiência, revisão sistemática ou integrativa, aceitos mas não publicados, e quando impossibilidade de acesso ao texto na íntegra. Realizou-se busca nas Bases de dados recomendadas para RS: US National Library of Medicine 
(PubMed), Portal da Biblioteca Virtual de Saúde (BVS), The Cochrane Central Register of Controlled Trials (CENTRAL) abrangendo a Base eletrônica editora Elsevier (EMBASE), Scientific Electronic Library Online (SciELO) e Health Evidence, esta última adotando-se 2 estratégias de busca para melhor abrangência.

\section{RESULTADOS}

\section{Seleção dos estudos}

Após a busca em bases de dados relevantes na área (DONATO H e DONATO M, 2019), a seleção de estudos identificou 256 artigos, sendo predominantemente em inglês (Figura 1).

Figura 1 - Diagrama do PRISMA - seleção de artigos

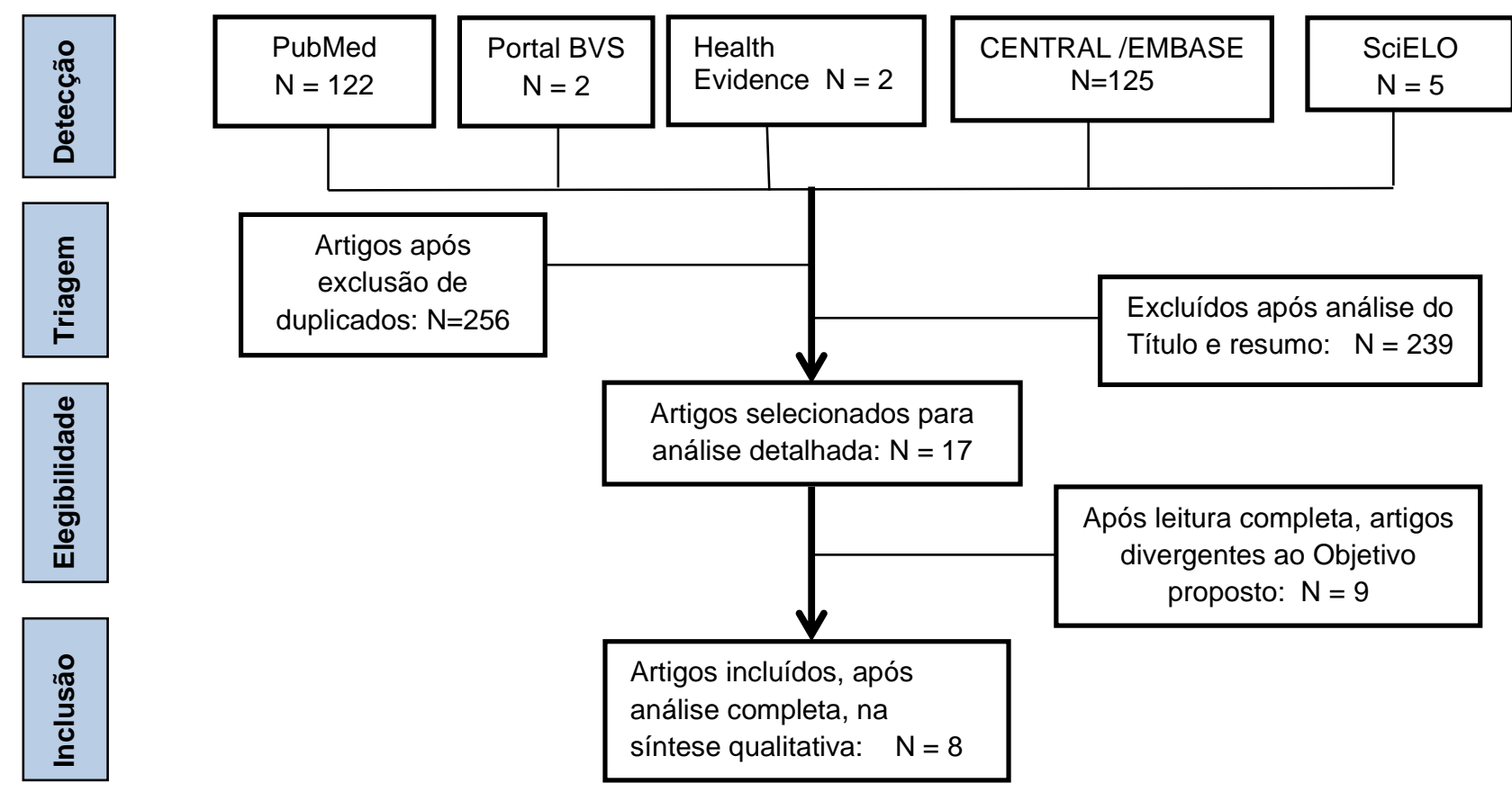

Fonte: Szpalher AS, 2019.

Não houve artigo duplicado. Após a seleção pela análise de títulos e resumo excluiu-se 239 artigos. 17 artigos foram analisados e, assim, 8 foram preservados para a síntese qualitativa e 9 foram excluídos; os motivos estão detalhados na Tabela 1.

Tabela 1 - Autores e motivo de exclusão dos artigos completos.

\begin{tabular}{cc}
\hline Salomé GM et al. (2015) & $\begin{array}{c}\text { Menciona distúrbios psicológicos no texto, não realiza esta abordagem. } \\
\text { Aborda a espiritualidade e religiosidade. }\end{array}$ \\
\hline Snyder RJ (2006) & Revisão de literatura \\
\hline Platsidaki E et al. (2017) & Revisão de literatura \\
\hline Norman D (2003) & Revisão de literatura \\
\hline Black AS et al. (1998) & $\begin{array}{c}\text { Estudo de prevalência sobre doenças crônicas, porém não } \\
\text { aborda doença venosa crônica ou UVP }\end{array}$ \\
\hline Jull A et al. (2017) & Analisa somente a qualidade de vida \\
\hline Romic I et al. (2015) & Analisa somente a qualidade de vida por meio de escalas validadas. \\
\hline Araújo RO et al. (2016) & Analisa somente a qualidade de vida \\
\hline Ahma SSK et al. (2016) & Analisa as complicações provenientes do pé diabético \\
\hline
\end{tabular}

Fonte: Szpalher AS, 2019. 


\section{Características dos estudos}

Foram obtidos estudos com desenhos diversos. Analisou-se um total de 816 voluntários, sendo 142 de grupo comparação. Houve diversidade quanto ao Tipo de estudo. Ressalta-se que, durante a busca, não foram encontrados artigos de antiga data. Os Desfechos apresentaram similaridade, nenhum apresentou ausência de sintomas/ transtornos psicológicos (Tabela 2).

Tabela 2 - Detalhamento dos estudos selecionados.

\begin{tabular}{|c|c|c|c|c|}
\hline Autor (es) & Tipo de estudo & Amostra & Instrumento & Desfecho \\
\hline $\begin{array}{l}\text { Mapplebeck } \\
\text { L (2008) }\end{array}$ & Estudo de caso & $\begin{array}{l}1 \text { mulher de } 42 \\
\text { anos com úlceras } \\
\text { ativas bilaterais }\end{array}$ & $\begin{array}{l}\text { Observação e } \\
\text { registro fotográfico }\end{array}$ & $\begin{array}{l}\text { Medo contínuo de necessitar realizar } \\
\text { amputação e diagnóstico de } \\
\text { depressão revertido após cicatrização } \\
\text { das feridas. Após cura das UVP, } \\
\text { houve resolução, também, no estado } \\
\text { psiquiátrico. }\end{array}$ \\
\hline $\begin{array}{l}\text { Jones JE et } \\
\text { al. (2008) }\end{array}$ & $\begin{array}{l}\text { Estudo de } \\
\text { prevalência }\end{array}$ & $\begin{array}{l}196 \text { indivíduos } \\
\text { portadores de } \\
\text { UVP }\end{array}$ & $\begin{array}{l}\text { Hospital Anxiety } \\
\text { and Depression } \\
\text { Scale (HADS) e } \\
\text { entrevista }\end{array}$ & $\begin{array}{l}\text { Odor e exsudato estão associados ao } \\
\text { alto score de depressão em } \mathrm{N}=52 \\
\text { (27\%) e ansiedade em } \mathrm{N}=50 \quad(26 \%) \text {. } \\
\text { Os entrevistados relatam limitação às } \\
\text { atividades e sintomas negativos. }\end{array}$ \\
\hline $\begin{array}{l}\text { Salomé GM } \\
\text { et al. (2012) }\end{array}$ & $\begin{array}{l}\text { Transversal } \\
\text { (observacional } \\
\text { analítico) }\end{array}$ & $\begin{array}{l}60 \text { pacientes de } \\
\text { um Ambulatório } \\
\text { de } \\
\text { feridas }\end{array}$ & $\begin{array}{l}\text { Entrevista com } \\
\text { Inventário de } \\
\text { Avaliação de } \\
\text { Depressão de Beck }\end{array}$ & $\begin{array}{l}91,66 \% \text { apresentou depressão, com } \\
\text { maior frequência de sintomas em } \\
\text { níveis leve a moderado ( } n=39 ; 65 \%) \text {, } \\
\text { além da depressão grave ( } n=9 ; 15 \%) \text {; } \\
\text { porém apresentou-se elevada em } \\
\text { todas as faixas etárias. }\end{array}$ \\
\hline $\begin{array}{l}\text { Barbosa } \\
\text { MLG et al. } \\
(2017)\end{array}$ & $\begin{array}{l}\text { Multicêntrico, } \\
\text { descritivo, } \\
\text { analítico, } \\
\text { prospectivo, } \\
\text { comparativo e } \\
\text { controlado }\end{array}$ & $\begin{array}{l}80 \text { portadores de } \\
\text { UVP na Atenção } \\
\text { básica: } \mathrm{N}=40 \\
\text { grupos controle } \\
\text { em atendimento } \\
\text { convencional e } \\
\mathrm{N}=40 \text { grupos } \\
\text { caso em } \\
\text { acupuntura }\end{array}$ & $\begin{array}{l}\text { Dados } \\
\text { relacionados à } \\
\text { lesão e Escala } \\
\text { HADS }\end{array}$ & $\begin{array}{l}\text { Grupo casos: média ansiedade em } 6 \\
\text { semanas: de } 8,25 \text { para } 4,43 \text {. Grupo } \\
\text { controle: média ansiedade em } 6 \\
\text { semanas: de } 12,70 \text { para } 5,00 \text {, ambos } \\
\text { aferidos em } 3 \text { momentos. Os } \\
\text { tratamentos resultaram em melhora de } \\
\text { ansiedade e depressão nos dois } \\
\text { grupos, com destaque o da } \\
\text { acupuntura. }\end{array}$ \\
\hline $\begin{array}{l}\text { Kouris A } \\
\text { et al. } \\
(2014)\end{array}$ & $\begin{array}{l}\text { Caso controle } \\
\text { (prospectivo } \\
\text { observacional) }\end{array}$ & $\begin{array}{l}102 \text { indivíduos em } \\
\text { tratamento de UVP, } \\
\text { arterial e mista e } 102 \\
\text { do grupo controle, no } \\
\text { ano de } 2012\end{array}$ & $\begin{array}{l}\text { Dermatology Life } \\
\text { Quality Index; HADS; } \\
\text { Rosenberg 's Self- } \\
\text { esteem Scale; } \\
\text { Loneliness Scale } \\
\end{array}$ & $\begin{array}{l}\text { Observou-se diferença estatística } \\
\text { significante nos scores de ansiedade } \\
\text { e depressão (HADS scale) e solidão } \\
\text { (UCLA scale) estando elevados em } \\
\text { comparação ao grupo controle. }\end{array}$ \\
\hline $\begin{array}{l}\text { Wachholz } \\
\text { PA et al. } \\
(2014)\end{array}$ & $\begin{array}{l}\text { Estudo } \\
\text { descritivo e } \\
\text { analítico, não } \\
\text { probabilístico }\end{array}$ & $\begin{array}{l}41 \text { portadores de } \\
\text { úlceras de diversas } \\
\text { etiologias, sendo a } \\
\text { UVP prevalente }\end{array}$ & $\begin{array}{l}\text { Dermatology Life } \\
\text { Quality Index (DLQI) }\end{array}$ & $\begin{array}{l}\text { Evidências de associação entre pior } \\
\text { percepção da QV e sintomas } \\
\text { depressivos; a severidade desses } \\
\text { sintomas foi uma das variáveis que } \\
\text { influenciaram no DLQI. }\end{array}$ \\
\hline $\begin{array}{l}\text { Walburn } \\
\text { J et al. } \\
(2017)\end{array}$ & $\begin{array}{l}\text { Caso controle } \\
\text { (prospectivo } \\
\text { observacional) }\end{array}$ & $\begin{array}{l}63 \text { indivíduos com } \\
\text { diagnóstico de UVP e } \\
\text { modelos efeito mistos } \\
\text { de controle de } \\
\text { variáveis }\end{array}$ & $\begin{array}{l}\text { Perceived Stress } \\
\text { Scale, HADS e } \\
\text { Illness Perception } \\
\text { Questionnaire }\end{array}$ & $\begin{array}{l}\text { O score sugeriu a presença de } \\
\text { ansiedade }(30,2 \%), \quad \text { depressão } \\
(22,2 \%) \text { e representação emocional } \\
\text { negativa (39\%) ao maior tempo de } \\
\text { cicatrização; com tempo de cura de } 22 \\
\text { semanas em comparação a } 11 \\
\text { semanas na representação menos } \\
\text { negativa. }\end{array}$ \\
\hline $\begin{array}{l}\text { Torres } \\
\text { SMSSO } \\
\text { et al. } \\
(2018)\end{array}$ & $\begin{array}{l}\text { Transversal } \\
\text { (cross } \\
\text { seccional) } \\
\text { comparativo }\end{array}$ & $\begin{array}{l}171 \text { indivíduos com } \\
\text { UVP da Atenção } \\
\text { Primária em } 1 \text { cidade } \\
\text { de Portugal e } 1 \text { do } \\
\text { Brasil }\end{array}$ & $\begin{array}{l}\text { Health-related quality } \\
\text { of life form (HRQOL) } \\
\text { e } \\
\text { Medical Outcomes } \\
\text { Study Item Short- } \\
\text { Form (SF-36). }\end{array}$ & $\begin{array}{l}\text { Em ambos os países houve } \\
\text { comprometimento no aspecto mental } \\
\text { e prejuízo emocional, ocasionado } \\
\text { aspecto como mudanças na imagem. } \\
\text { O resultado foi melhor no Brasi } \\
\text { (mediana 68) do que em Portugal } \\
\text { (mediana } 88 \text { ) }\end{array}$ \\
\hline
\end{tabular}

Fonte: Szpalher AS, 2019. 
Para a análise de viés e qualidade foi utilizada a NEWCASTLE - OTTAWA Quality Assessment Scale Case Control Studies (NOS), conforme recomendação PRISMA. Estudos sem grupo controle foram incluídos, intencionando-se expandir a busca diante de resultados limitados (Tabela $\mathbf{3}$ ).

Tabela 3 - Escala de Newcastle: Case control studies.

\begin{tabular}{|c|c|c|c|}
\hline Parâmetros: Seleção / Comparabilidade / Desfecho & $\begin{array}{l}\text { Kouris A et al. } \\
(2014)\end{array}$ & $\begin{array}{l}\text { Walburn J et } \\
\text { al. (2017) }\end{array}$ & $\begin{array}{l}\text { Barbosa MLG et } \\
\text { al. (2017) }\end{array}$ \\
\hline A definição do caso é adequada & $*$ & $*$ & $*$ \\
\hline Representatividade dos casos & * & * & $*$ \\
\hline Seleção do grupo controle & $*$ & & $*$ \\
\hline Definição de controle & * & $*$ & * \\
\hline Comparação do caso e controle no desenho ou na análise & $*$ & $*$ & $*$ \\
\hline Determinação da exposição & $*$ & * & \\
\hline Mesmo método de determinação de casos e de controle & * & & * \\
\hline Taxa de não resposta & * & * & \\
\hline
\end{tabular}

Fonte: http://www.ohri.ca/programs/clinical_epidemiology/oxford.asp. Tabela elaborada por Szpalher AS, 2019.

\section{Síntese dos resultados}

Foi observada homogeneidade com relação ao perfil das amostras, evidenciando que os sintomas psicológicos são um evento já esperado no público estudado. Diversas escalas foram utilizadas, porém, todas estas aferem dimensões semelhantes, com destaque para o domínio psicológico e bem-estar. A diversidade de locais nos quais os estudos foram empregados refletem a semelhança no sofrimento vivenciado por estes indivíduos, independente do país no qual habitam. Segundo Walburn J et al. (2017) a HADS tem validade e confiabilidade alta, sendo instrumento de escolha pelos autores.

Assim, todos os sintomas psicológicos manifestados pelos portadores de UVP, apresentados nos estudos, estão expostos no Quadro 2.

Quadro 2 - Síntese dos achados e frequência de aparecimento nos estudos (N)

\begin{tabular}{ll}
\hline Depressão / Isolamento social / Ansiedade & 8 \\
\hline Baixa autoestima / Autoaversão / Raiva / Inibição para o trabalho / Retração social / privação do sono & 3 \\
\hline Alterações na saúde física / Sentimento de desgosto / Vergonha de si mesmo e das pessoas / Sentimento \\
de desqualificação para as atividades / Perda da feminilidade / Pessimismo / distorção da imagem corporal & 2 \\
\hline Ideia suicida / Preocupação somática / Autodepreciação / Fadiga / Falta de satisfação / Sensação de culpa; & 1 \\
de punição; de fracasso; de estar mutilado / Perda de apetite / Crise de choro / Perda de peso &
\end{tabular}

Fonte: Szpalher AS, 2019.

\section{DISCUSSÃO}

Mapplebeck L (2008) acompanha, em seu estudo, a jornada de convivência de uma mulher com UVP bilateral, extensa e de difícil tratamento. Porém, ressalta que após adequada conduta clínica e manejo sensível das lesões, obteve-se a completa cura das UVP. A resolução deste problema trouxe a esta paciente o sentimento de controle da própria vida, perspectiva positiva com relação ao futuro, melhora da relação na rotina conjugal e suspensão médica do antipsicótico com função antidepressiva e otimismo para aderir uma dieta para perda de peso.

Segundo Jones JE et al. (2008), as pessoas que experimentam o primeiro episódio de ulceração, geralmente com exsudação considerável, vivenciam um impacto devastador na vida cotidiana. Além disso, a presença de odor é o fator chave associado à alta prevalência de depressão; provoca uma resposta emocional e psicológica negativas como vergonha, nojo, aversão de si próprio e o sentimento de sentir-se insuportável por tornar-se uma pessoa frustrada e zangada. Na exposição das entrevistas, há relatos de grande desânimo, limitação diária e de comparar o odor a um rato ou pessoa morta dentro de casa. 
Salomé GM et al. (2012) citam a aposentadoria por invalidez como uma consequência da UVP. Ainda encontraram diferença estatística relacionada à presença de UVP e idade em pacientes com sintomas depressivos, sendo que os transtornos estão mais presentes, notadamente, em pacientes idosos. Por meio do Inventário de Avaliação de Depressão de Beck, concluiu-se que portadores de UVP apresentam diversos níveis de sintomas depressivos, incluindo o nível grave; e relacionam este resultado ao sentimento de medo de sofrer em decorrência da presença da lesão de pele. Apesar de diversos estudos apresentarem maior grau de depressão em mulheres, este estudo não obteve significante diferença entre gêneros.

Barbosa MLG et al. (2017) ressaltam a importância de conhecer o grau de escolaridade do indivíduo, de modo a planejar adequadamente os cuidados com a UVP, afim de facilitar a compreensão acerca da prescrição do Enfermeiro, e ressaltam a presença de sinais de transtornos psicológicos como a sensação de mutilação pela qual o indivíduo passa, de modo a dificultar, inclusive, momentos corriqueiros como o ato de vestir-se.

Vale destacar outra importante conclusão do estudo de Kouris A et al. (2014): o grupo feminino apresentou scores mais elevados em comparação ao grupo masculino nas escalas de solidão (UCLA), autoestima (RSES) e subescala de ansiedade (HADS-A). Este resultado corrobora com estudos anteriores; que é explicado pelo fato de as mulheres deixarem as pernas mais expostas, tornando as lesões visíveis e, consequentemente, causando sentimento de perda da feminilidade e de ser pouco atraente. A mobilidade comprometida causada pela UVP traz de inutilidade, provavelmente pela limitação em realizar as tarefas socialmente tradicionais da mulher, como cuidar do lar e dos filhos.

Segundo Kouris A et al. (2014), indivíduos com UVP precisam vivenciar a importante adaptação ao seu problema, utilizando, assim, diferentes estratégias de enfrentamento para lidar com a doença. Ainda recomendam uma abordagem integral a esses indivíduos, que inclua apoio psicológico e social, prevenção do isolamento e produção de oportunidades para compartilhamento de experiências comuns. Wachholz PA et al. (2014) citam outras variáveis que influenciaram o resultado do Dermatology Quality of Life Index (DLQI): etiologia da lesão, dor relacionada à UVP e tempo de início. Identificar os sintomas depressivos e sua gravidade justifica a necessidade de novas pesquisas que confirmem a relação entre estes sintomas e a QV de portadores de UVP. Felizmente, o estudo encontrou uma alta proporção de independência funcional, mesmo a amostra apresentando idade média de 61,78 anos.

Walbum J et al. (2017) avaliaram as características, duração, e mensuração das UVP durante 24 semanas. Após aplicação das escalas mencionadas, observaram que $30,4 \%$ das UVP mantiveram-se abertas na presença de níveis elevados de estresse e depressão e de percepção negativa da doença (IP). Esse resultado, em comparação aos modelos de variáveis controladas, foi associado a uma taxa significativamente mais lenta de melhora da UVP, independente do tamanho desta. Concluem que é necessário garantir que pacientes entendam a sua condição psicológica e física, e aceitam recomendações.

Torres SMSSO et al. (2018) reconhecem a urgência em adaptar o cuidado de saúde ao meio social proporcionando melhores condições. Ainda alertam sobre a necessidade de se ter compreensão por parte da equipe de saúde, auxiliando, assim, a promover assistência adequada ao portador de UVP. Para complementar, Saraiva DMRF et al. (2013) abrangem as consequências da UVP ao mencionar o aumento do tempo de hospitalização, e comenta o prejuízo causado ao domínio psicológico, tanto do indivíduo quanto dos familiares, podendo interferir na maneira como o indivíduo se percebe. Estudos diversos relatam associação entre estresse e cicatrização retardada.

\section{CONSIDERAÇÕES FINAIS}

Diante da pouca produção relacionada à temática, sugerem-se estudos que aprofundem o impacto dos transtornos psicológicos nas diversas fases de UVP e como estes transtornos afetam o tratamento em nível citológico e histológico, principalmente da rede venosa superficial e profunda. Estudos nesse sentido podem trazer maiores reflexões acerca da importância do atendimento psicológico e psiquiátrico, em conjunto com atividades promotoras de bem-estar e de manutenção do convívio social. Em outras palavras, vê-se a necessidade em realizar aconselhamento em saúde mental, com abordagem holística, para enfrentar as múltiplas necessidades psicossociais complexas inerentes à UVP. 


\section{REFERÊNCIAS}

1. ARAÚJO RO, et al. Impacto de úlceras venosas na qualidade de vida de indivíduos atendidos na atenção primária. Aquichan, 2016; 16(1): 56-66.

2. BARBOSA MLG et al. Avaliação da ansiedade e da depressão em pacientes com úlcera venosa tratados com acupuntura. Rev Enferm UFPE, 2017; 11(9): 3574-3582.

3. DONATO H, DONATO M. Etapas na Condução de uma Revisão Sistemática. Acta Med Port, 2019; 32(3): $227-235$.

4. GALVÃO TF, PANSANI TSA. Principais itens para relatar Revisões sistemáticas e Meta-análises: A recomendação PRISMA. Epidemiol. Serv. Saúde, 2015; 24(2): 335-342.

5. JONES JE et al. Impact of exudate and odour from chronic venous leg ulceration. Nursing Standard, 2008; 22(45): 5361.

6. KOURIS A, et al. Quality of life psychosocial characteristics in Greek patients with leg ulcers: a case control study. International Wound Journal, 2014; $1-4$.

7. MAPPLEBECK L. Case study: psycosocial aspects of chronic bilateral venous leg ulcers. British Journal of Community Nursing, 2008, 33-37.

8. PLATSIDAKI E, et al. Psychosocial aspects in patients with chronic leg ulcers. Wounds, 2017; 29(10): $306-310$.

9. ROMIC I, et al. Quality of life and perception of disease in patients with chronic leg ulcer. Acta Clin Croat, 2015; 54(3): 309-314.

10. SALOMÉ GM, et al. Avaliação de sintomas depressivos em pessoas com úlcera venosa. Rev Bras Cir Plást, 2012; 27(1): 124-129.

11. SALOMÉ GM, et al. Association of sociodemographic factors with hope for cure, religiosity, and spirituality in patients with venous ulcers. Wound Care Journal, 2015; 28(2): 76-82.

12. SARAIVA DMRF, et al. Qualidade de vida do utente com úlcera venosa crônica. Revista de Enfermagem Referência, 2013; 3(10): 109-118.

13. TORRES SMSSO, et al. Health-related quality of life in patients with venous leg ulcer treated in primary care in Brazil and Portugal, 2018; Plos One,13(4):1-10.

14. WACHHOLZ PA, et al. Quality of life profile and correlated factors in chronic leg ulcer patients in the mid-west of São Paulo State, Brazil. A Bras Dermatol, 2014; 89(1): 73-81.

15. WALBUM J, et al. Stress, Illness Perceptions, Behaviors, and Healing in Venous Leg Ulcers: Findings from a Prospective Observational Study. Psychosom Med, 2017; 79(5): 585-592. 